\title{
Feasibility and correlation of standard 2D speckle tracking echocardiography and automated function imaging derived parameters of left ventricular function during dobutamine stress test
}

\author{
Karina Wierzbowska-Drabik $\cdot$ Piotr Hamala $\cdot$ \\ Nikolina Roszczyk • Piotr Lipiec • Michał Plewka • \\ Radosław Kręcki · Jarosław Damian Kasprzak \\ Received: 11 December 2013/Accepted: 3 February 2014/Published online: 13 February 2014 \\ (c) The Author(s) 2014. This article is published with open access at Springerlink.com
}

\begin{abstract}
Speckle tracking echocardiography (STE) is a method of quantitative assessment of myocardial function complementary to ejection fraction and visual evaluation. Standard STE analysis, demands manual tracing of the myocardium whereas automated function imaging (AFI) offers more convenient (based on selection of three points) assessment of longitudinal strain. Nevertheless, feasibility and correlation between both methods were not thoroughly examined, especially during tachycardia at peak stage of dobutamine stress echocardiography (DSE). We performed DSE in 238 patients (pts) with recording of apical views during baseline (0) and peak (1) DSE and analyzed them by STE and AFI. According to angiography, 127/238 pts had significant $(\geq 70 \%)$ lesions in coronary arteries. We assessed correlations between STE and AFI derived peak systolic longitudinal strain values for global and regional parameters, feasibility, time of analysis and interobserver agreement. Global systolic longitudinal strain measured during baseline and peak stage of DSE by AFI showed very good correlation with standard STE parameters, with correlation coefficients $r=0.90$ and $r=0.86$ respectively $(p<0.0001)$. For regional parameters correlation coefficients ranged from 0.83 to 0.85 for baseline and from 0.70 to 0.79 for peak DSE. Both methods provided good and similar feasibility with only $1 \%$ segments excluded from analysis at peak stage of DSE with shorter time and lower coefficient of variance offered by AFI. Global and regional longitudinal strain achieved by faster and less operatordependent AFI method correlate well with standard more
\end{abstract}

K. Wierzbowska-Drabik ( $)$ - P. Hamala · N. Roszczyk P. Lipiec - M. Plewka · R. Kręcki - J. D. Kasprzak

Chair and Department of Cardiology, Medical University of Lodz, Kniaziewicza 1/5, 91-347 Lodz, Poland

e-mail: wierzbowska@ptkardio.pl time-consuming STE analysis during baseline and peak stage of DSE.

Keywords Dobutamine stress echocardiography ·

Speckle tracking - Automated function imaging - Systolic longitudinal strain

\section{Introduction}

Stress echocardiography is, besides nuclear imaging, one of the leading noninvasive tests widely applied in the diagnosis of coronary artery disease $[1,2]$. The proven diagnostic advantage over ECG-based detection of ischemia, observed especially in women, is however, limited by subjectivity of visual assessment of regional contractile function. Thus, various methods potentially increasing interobserver agreement and providing quantitative parameters of segmental systolic function are intensely tested [3-8]. The clinical introduction of noninvasive tools for the assessment of myocardial deformation provided the impulse to testing of their utility in the field of stress echocardiography [9]. Since early 90, deformation imaging based on Doppler method has been tested, showing the relationship between ischemia and longitudinal strain rate decrease and discovering the phenomenon of post systolic shortening with possibility of its quantification [10-13]. The application of two-dimensional image (2D)-based speckle tracking echocardiography (STE) allowed to overcome some of drawbacks of Doppler-derived method such as angle-dependency, the general limitation to longitudinal direction of deformation and the lack of possibility of accurate evaluation of the left ventricular apex at the cost of lower time resolution of STE [14]. In spite of wider application and more user-friendly analysis of myocardial 
deformation with standard STE method, quantitative assessment of regional left ventricular function is still timeconsuming and the presentation of results lacks synthetic display. These shortcomings are especially important for the assessment of myocardial function during dobutamine stress echocardiography (DSE) when meticulous evaluation of segmental function should be repeated at least at baseline and peak stage. Thus a new based on STE technique, automated function imaging (AFI) allowing a faster assessment of regional longitudinal strain of the left ventricle and clear results presentation as a polar map has recently gained wide interest and has been tested in various clinical settings although its applicability for DSE is still poorly documented. The aim of our study was to evaluate the relationships of regional and global parameters of left ventricular longitudinal strain derived by standard STE and a novel, semiautomatic AFI method, during baseline and peak stage of DSE and compare feasibility of both methods.

\section{Methods}

Study group and protocol

We examined 238 subjects (105 women, mean age $62 \pm 9$, mean left ventricle ejection fraction $59 \pm 10 \%$ ) with symptoms suggesting stable angina and scheduled for myocardial ischemia testing. The study protocol encompassed: medical history, physical examination, resting ECG and laboratory data (lipoproteins, glucose, and creatinine), transthoracic echocardiographic examination, dobutamine stress echocardiography with early atropine administration (DSE) and angiographic examination in all patients performed no later than 3 months after DSE. The assessment of coronary arteries was done by invasive coronary angiography in 208 patients and by computed tomography in 35 subjects ( 5 patients had both examinations). As significant lesion stenosis $\geq 50 \%$ for left main coronary artery (LMCA) and $\geq 70 \%$ for others epicardial arteries was defined. All study patients were in sinus rhythm and had no significant valve disease (except for mild to moderate insufficiency). Exclusion criteria included contraindications to dobutamine and atropine: acute coronary syndromes, valve or outflow tract stenosis, blood pressure $>200 / 100 \mathrm{mmHg}$, ventricular tachycardia, glaucoma or prostatic adenoma. Demographic characteristics and echocardiographic data of the examined subjects are displayed in Tables 1 and 2. All subjects gave written informed consent to participate in the study and the protocol was approved by Ethical Commission of Medical
Table 1 Demography, risk factors and treatment of the studied group

\begin{tabular}{lll}
\hline Parameter in studied group $\mathrm{n}=238$ & Mean \pm SD & Range \\
\hline Age (years) & $62 \pm 9$ & $38-61$ \\
Height (cm) & $168 \pm 9$ & $146-187$ \\
Body mass (kg) & $81 \pm 15$ & $43-125$ \\
Waist to hip ratio & $0.92 \pm 0.1$ & $0.64-1.2$ \\
Body mass index $\left(\mathrm{kg} / \mathrm{m}^{2}\right.$ ) & $28.7 \pm 4.7$ & $17.6-46.9$ \\
Body surface area (m $\left.{ }^{2}\right)$ & $1.94 \pm 0.2$ & $1.35-2.52$ \\
Hypertension (number \%) & 207 & 86 \\
Diabetes mellitus (number \%) & 66 & 28 \\
Smoking (number \%) & 135 & 56 \\
Hypercholesterolemia (number \%) & 197 & 82 \\
Hypertriglyceridemia (number \%) & 152 & 63 \\
Family history of CAD (number \%) & 41 & 17 \\
History of myocardial infarction & 70 & 29 \\
$\quad$ (number \%) & & \\
Acetylsalicylic acid (number \%) & 208 & 87 \\
Clopidogrel (number \%) & 75 & 32 \\
Betaadrenolytic (number \%) & 177 & 74 \\
ACE inhibitor (number \%) & 183 & 76 \\
Statin (number \%) & 204 & 86 \\
Long-acting nitrates (number \%) & 116 & 49 \\
\hline CAD con & & \\
\hline
\end{tabular}

$C A D$ coronary artery disease, $A C E$ angiotensin-converting enzyme, $L D L$ low density lipoprotein, $H D L$ high density lipoprotein

University of Lodz (number of agreement RNN/119/10 KE from 13.07.2010).

\section{Echocardiography at rest and during DASE}

Transthoracic echocardiography was performed with VIVID 7 Dimension (GE Vingmed Ultrasound AS, Horten, Norway) using M4S probe in harmonic mode $2.0 / 4.3 \mathrm{MHz}$ with maximal frame per second (FPS) count available at necessary sector width. Range of FPS was from 64 to 112 with mean value 83 . The assessment of left ventricular (LV) systolic and diastolic volumes and ejection fraction was done using a modified Simpson's method from triplane view obtained with volumetric probe $3 \mathrm{~V}$. Mass of LV was calculated from Devereaux's formula with measurements of LV walls and cavity was performed according to Penn convention. Wall thickness and chamber dimensions were measured from 2D imaging in parasternal long axis view. The echocardiographic measurements were done following American Society of Echocardiography/European Association of Echocardiography guidelines. The assessment of left ventricular contractility was done visually by two experts and classified for each segment as norm kinesis, hypokinesis, akinesis or diakinesis. 18-segments model of left ventricle was applied dividing each of $\mathrm{LV}$ wall 
Table 2 Characteristic of basic echocardiographic parameters of studied group at baseline

\begin{tabular}{lcr}
\hline Parameter in studied group $\mathrm{n}=238$ & Mean \pm SD & Range \\
\hline LVd (mm) & $47 \pm 5$ & $35-61$ \\
LVs $(\mathrm{mm})$ & $33 \pm 5$ & $22-50$ \\
PWd (mm) & $11 \pm 1$ & $8-16$ \\
PWs $(\mathrm{mm})$ & $14 \pm 2$ & $10-19$ \\
IVSd (mm) & $12 \pm 2$ & $8-17$ \\
IVSs $(\mathrm{mm})$ & $15 \pm 2$ & $11-20$ \\
Ao $(\mathrm{mm})$ & $33 \pm 4$ & $25-46$ \\
LA $(\mathrm{mm})$ & $40 \pm 4$ & $30-53$ \\
RV $(\mathrm{mm})$ & $26 \pm 2$ & $20-32$ \\
E/A & $0.89 \pm 0.37$ & $0.4-4.0$ \\
LV mass $(\mathrm{g})$ & $240 \pm 66$ & $102-464$ \\
LV mass index $\left(\mathrm{g} / \mathrm{m}^{2}\right)$ & $123 \pm 29$ & $51-254$ \\
\hline
\end{tabular}

$L V d$ left ventricular diastolic dimension, $L V s$ left ventricular systolic dimension, $P W d$ diastolic thickness of the left ventricular posterior wall, $P W s$ systolic thickness of the left ventricular posterior wall, IVSd diastolic thickness of left ventricular septum, IVSS systolic thickness of left ventricular septum, Ao aortic dimension, $L A$ left atrial dimension, $R V$ right ventricular dimension, $E / A$ ratio of early to atrial mitral inflow peak velocity, $L V$ mass left ventricular mass, $L V$ mass index left ventricular mass index

$* p<0.05$

visualized from apical projections into three segments: basal, mid and apical. The worsening of contractility in at least two adjacent segments of left ventricle was consistent with a positive dobutamine stress test. Early atropine administration protocol was applied. Dobutamine was administered in the intravenous infusion in doses of 10, 20, 30 and $40 \mu \mathrm{g} / \mathrm{kg} / \mathrm{min}$ during 3-min stages whereas atropine was added in $0.5 \mathrm{mg}$ fractional doses after the second stage of infusion up to the total dose of $2 \mathrm{mg}$. The infusion of dobutamine was stopped when age-adjusted HR limit, positive stress test result or other criteria for terminating DSE were fulfilled. For safety reasons, the presence of minimum two persons during each study was mandatory, $\mathrm{BP}$ was measured during each stage of test, 12-lead ECG was recorded immediately after stopping dobutamine infusion and a prolonged observation up to $1 \mathrm{~h}$ after the test was scheduled.

The assessment of deformation

Digital loops from standard echocardiographic views (three apical views and LV short axis views at three levels- mitral valve, papillary muscle and apical) were digitally stored at baseline and peak stage of the infusion for further analysis. The calculation of regional and global parameters of deformation was done off-line using EchoPac 6.1.0 workstation (GE Vingmed Ultrasound). The allocation of regional values of longitudinal strain by standard 2D speckle tracking imaging required manual marking of endocardial contour and adjustment of the region of interest to encompass the myocardial wall in all three apical views. For the analysis, we assigned left ventricular segments with consecutive numbers in clockwise order

Table 3 Comparison of heart rate, blood pressure and chosen echocardiographic parameters of studied group between baseline and peak stage of DSE

\begin{tabular}{|c|c|c|c|}
\hline Parameter in studied group $n=238$ & Mean \pm SD at baseline & Mean \pm SD at peak & $p$ value \\
\hline Heart rate DSE (bpm) & $66 \pm 10$ & $139 \pm 17$ & $<0.0001$ \\
\hline Blood pressure systolic $(\mathrm{mmHg})$ & $129 \pm 18$ & $142 \pm 25$ & $<0.0001$ \\
\hline Blood pressure diastolic $(\mathrm{mmHg})$ & $71+10$ & $77 \pm 16$ & $<0.0001$ \\
\hline $\mathrm{EF}(\%)$ & $58 \pm 10$ & $66 \pm 9$ & $<0.0001$ \\
\hline $\mathrm{S}^{\prime}$ lat $(\mathrm{cm} / \mathrm{s})$ & $8 \pm 2$ & $14 \pm 5$ & $<0.0001$ \\
\hline $\mathrm{E}^{\prime}$ lat $(\mathrm{cm} / \mathrm{s})$ & $10 \pm 3$ & $15 \pm 4$ & $<0.0001$ \\
\hline Wall motion index & $1.08 \pm 0.24$ & $1.17 \pm 0.27$ & $<0.0001$ \\
\hline Global SLS by STE (\%) & $-16.4 \pm 3.6$ & $-15.0 \pm 4.1$ & $<0.0001$ \\
\hline Global SLS by AFI (\%) & $-17.4 \pm 3.9$ & $-16.6 \pm 4.3$ & $=0.0003$ \\
\hline Average SLS 2 ch by STE (\%) & $-16.3 \pm 4.2$ & $-14.9 \pm 4.8$ & $<0.0001$ \\
\hline Average SLS 3 ch by STE (\%) & $-16.3 \pm 4.1$ & $-14.7 \pm 4.9$ & $<0.0001$ \\
\hline Average SLS 4 ch by STE (\%) & $-16.6 \pm 4.0$ & $-15.4 \pm 5.2$ & $=0.0001$ \\
\hline Average SLS 2 ch by AFI (\%) & $-17.6 \pm 4.4$ & $-16.2 \pm 5.0$ & $<0.0001$ \\
\hline Average SLS 3 ch by AFI ( $\%)$ & $-17.5 \pm 4.6$ & $-16.9 \pm 5.4$ & $=0.0536$ \\
\hline Average SLS 4 ch by AFI (\%) & $-17.1 \pm 4.3$ & $-16.5 \pm 5.1$ & $=0.0328$ \\
\hline
\end{tabular}

$\overline{D S E}$ dobutamine stress echocardiography, $L V E D V$ end diastolic volume of left ventricle, $L V E S V$ end systolic volume of left ventricle, $E F$ ejection fraction, $S^{\prime}$ lat peak velocity of lateral part of mitral annulus in systolic phase, $E^{\prime}$ lat peak velocity of lateral part of mitral annulus in diastolic phase, SLS systolic longitudinal strain, STE speckle tracking echocardiography, AFI automated function imaging, 2 ch apical twochamber view, 3 ch apical three-chamber view, 4 ch apical four-chamber view 

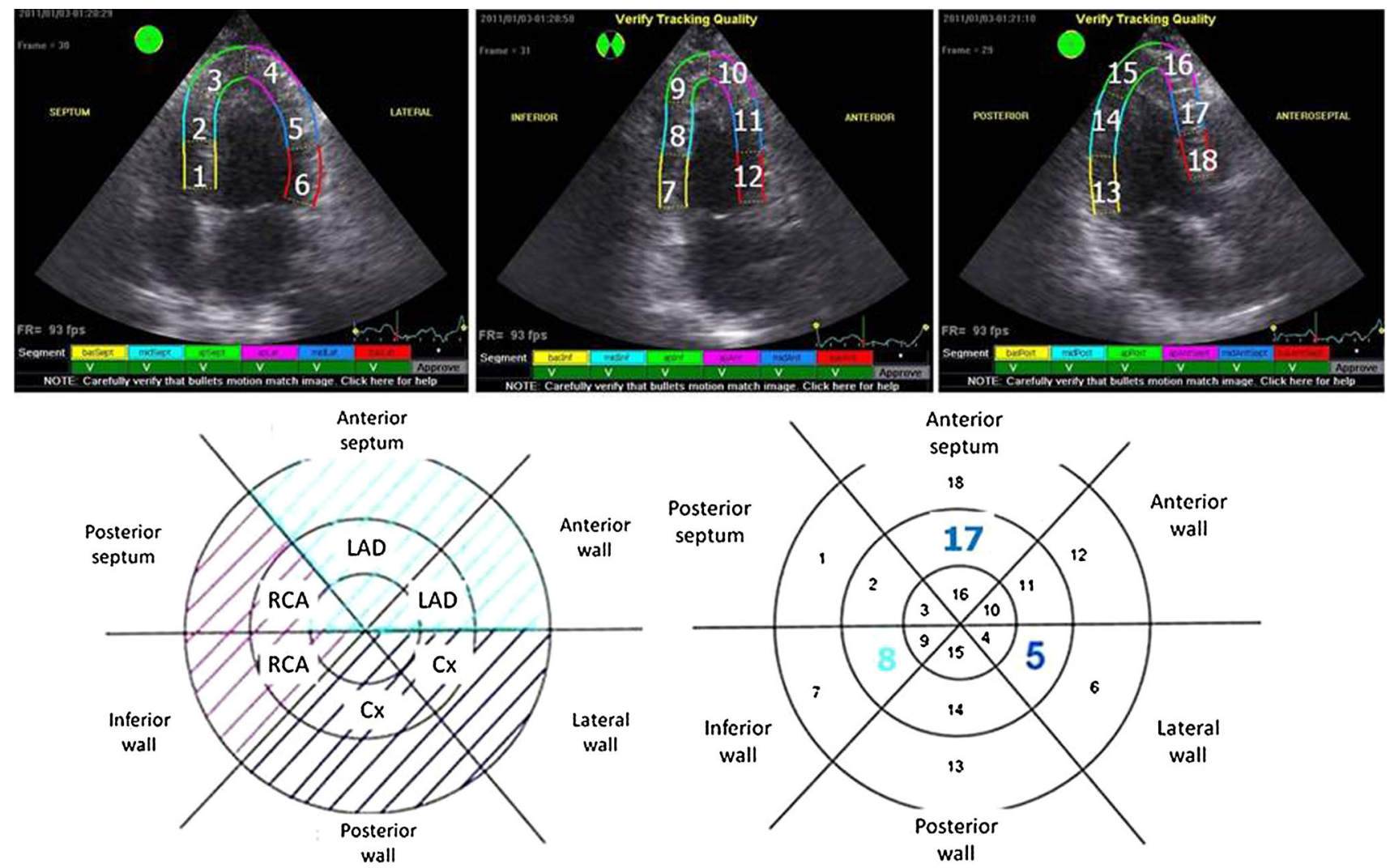

Fig. 1 The annotation of the left ventricular segments and definition of regions supplied by respective coronary arteries

starting from basal septum in 4-chamber view, through 2-chamber to 3-chamber view. Mid-segments from lateral wall, inferior wall and anterior septum were chosen as marker segments for the circumflex, right coronary artery and left anterior descending artery territories respectively. The annotation of the myocardial segments and definition of region supplied by respective coronary arteries are displayed in Fig. 1. For each LV segment the value of peak systolic longitudinal strain was measured at baseline and peak level of dobutamine stress test SLS $_{0}$ and SLS $_{1}$ respectively, systolic longitudinal strain). Additionally, the averaged values of SLS for each apical view were measured and global SLS from 18 left ventricle segments was calculated. The obtaining of regional and global systolic longitudinal strain by AFI method demanded manual marking of three points (two on basal and one on apical endocardium) in each apical view and accepting the resulting region of interest. The rounded segmental values of peak systolic longitudinal strain were displayed in a straightforward format of polar map with additional information regarding the averaged (obtained from six segments) and global (obtained from 18 segments) systolic longitudinal strain.

The parameters measured by both techniques were defined as the peak deformation in longitudinal direction achieved for assessed segment during systole, before the aortic valve closure. Correlations were calculated for global and regional longitudinal strain in all segments.

\section{Statistical analysis}

Statistical analysis was performed using MedCalc V. 12.1.4. (Frank Schoonjans Belgium). Continuous variables were expressed as means and standard deviations. Mean values were compared with $t$ Student test for paired variables. Chi square test was used to test the dichotomous variables distribution. For comparison of parameters obtained by STE and AFI Pearson correlation coefficients were calculated and Bland-Altman analysis performed. For interobserver variability assessment we calculated coefficients of variance. Duration of STE and AFI calculations and interobserver agreement were assessed in the group of 12 randomly selected subjects.

\section{Results}

Time needed to analyze and obtain regional, averaged (from six segments) and global (from 18 segments) systolic longitudinal strain by classic STE was $367 \pm 39$ s and by 
Table 4 The feasibility of longitudinal strain analysis estimation by STE and AFI in all left ventricular segments at rest and peak stage of DSE

$S T E_{0}$ feasibility of strain measurements done by STE at rest, $A F I_{O}$ feasibility of strain measurements done by AFI at rest, $S T E_{1}$ feasibility of strain measurements done by STE at peak stress test, $A F I_{I}$ feasibility of strain measurements done by AFI at peak stress test

\begin{tabular}{lllll}
\hline Segment & $\mathrm{STE}_{0}(\%)$ & $\mathrm{AFI}_{0}(\%)$ & $\mathrm{STE}_{1}(\%)$ & $\mathrm{AFI}_{1}(\%)$ \\
\hline Basal posterior septum (seg. 1) & 100 & 100 & 100 & 99.6 \\
Mid posterior septum (seg. 2) & 100 & 100 & 100 & 100 \\
Apical posterior septum (seg. 3) & 100 & 100 & 100 & 100 \\
Apical lateral wall (seg. 4) & 100 & 100 & 99.6 & 100 \\
Mid lateral wall (seg. 5) & 99.2 & 98.7 & 97.1 & 97.9 \\
Basal lateral wall (seg. 6) & 97.9 & 98.3 & 94.5 & 95 \\
Basal inferior wall (seg. 7) & 100 & 100 & 99.6 & 98.7 \\
Mid inferior wall (seg. 8) & 100 & 100 & 100 & 100 \\
Apical inferior wall (seg. 9) & 99.6 & 100 & 100 & 100 \\
Apical anterior wall (seg. 10) & 99.6 & 99.6 & 100 & 100 \\
Mid anterior wall (seg. 11) & 99.6 & 99.6 & 99.2 & 98.7 \\
Basal anterior wall (seg. 12) & 100 & 99.2 & 97.1 & 96.2 \\
Basal posterior wall (seg. 13) & 100 & 99.2 & 98.3 & 96.6 \\
Mid posterior wall (seg. 14) & 100 & 100 & 99.2 & 98.7 \\
Apical posterior wall (seg. 15) & 100 & 100 & 100 & 99.6 \\
Apical anterior septum (seg. 16) & 100 & 99.2 & 99.6 & 100 \\
Mid anterior septum (seg. 17) & 100 & 99.6 & 100 & 100 \\
Basal anterior septum (seg. 18) & 99.6 & 99.2 & 98.7 & 99.6 \\
\hline
\end{tabular}

AFI $168 \pm 28 \mathrm{~s}$, the difference was statistically significant with $p<0.0001$. Interobserver variability (coefficient of variance) calculated for regional longitudinal strain in segment number one (basal septum) was 8.7 and $16 \%$ respectively for AFI at baseline and peak stage of DSE and for STE 13.3 and $24.2 \%$.

To our study we included only subjects $(n=238)$ in which visual assessment of endocardium was feasible in all left ventricular segments $(n=4,284$ segments $)$ we excluded-six patients $(2.5 \%)$ from the study because of insufficient visualization. Suboptimal quality of 2D image-as assessed automatically by software despite manual correction of region of interest- led to exclusion of 11 segments $(0.26 \%)$ from STE and 18 segments $(0.42 \%)$ from AFI analysis at rest. During the peak stage of DSE 41 segments $(0.96 \%)$ were suboptimal for STE analysis and $46(1.07 \%)$ were suboptimal for AFI quantification and excluded from the analysis. For both methods proportion of segments with suboptimal quality was significantly higher at peak stage of stress test than at baseline $(p=0.0001$ for STE and $p=0.0007$ for AFI) whereas the differences of feasibility between STE and AFI respectively at baseline and stress were not significant.

The feasibility of longitudinal systolic strain analysis at rest and stress calculated for individual left ventricular segments (range from 94.5 to $100 \%$ ) is displayed in Table 4. Figure 2 presents numbers and localization of excluded segments in polar maps of the left ventricle.

Expectedly, dobutamine induced the increase of heart rate, blood pressure, ejection fraction and peak velocities of mitral annulus as summarized in Table 3 . The diastolic and systolic volumes of left ventricle and absolute values of longitudinal strain decreased during dobutamine stress test, see Table 3. Global systolic longitudinal strain measured during baseline and peak stage of DSE by AFI showed strong correlation with standard STE parameters (correlation coefficients $\mathrm{r}=0.90$ and $\mathrm{r}=\mathrm{v} 86$ respectively, $p<0.0001$ ), see Fig. 3. The good agreement of the examined methods for determination of global strain was also confirmed in Bland-Altman analysis, see Fig. 4.

The comparison of regional deformation parameters measured in subsequent left ventricular segments indicated highly significant linear relationship of values from both methods ( $p<0.0001)$, although correlation coefficients for measurements performed at baseline were slightly higher than during peak stage of dobutamine test. In the Fig. 5 the relationships between systolic longitudinal strain measured by STE and AFI method in three mid-ventricular "marker" segments supplied by left anterior descending, circumflex and right coronary artery, respectively are shown.

\section{Discussion}

Although the application of two-dimensional speckle-tracking in the clinical studies is reaching the decade, and the newest techniques concerns three-dimensional and layerspecific assessment of myocardial function there is still a paucity of data comparing standard 2D STE and its newer modification AFI in the same subset of patients, especially in 

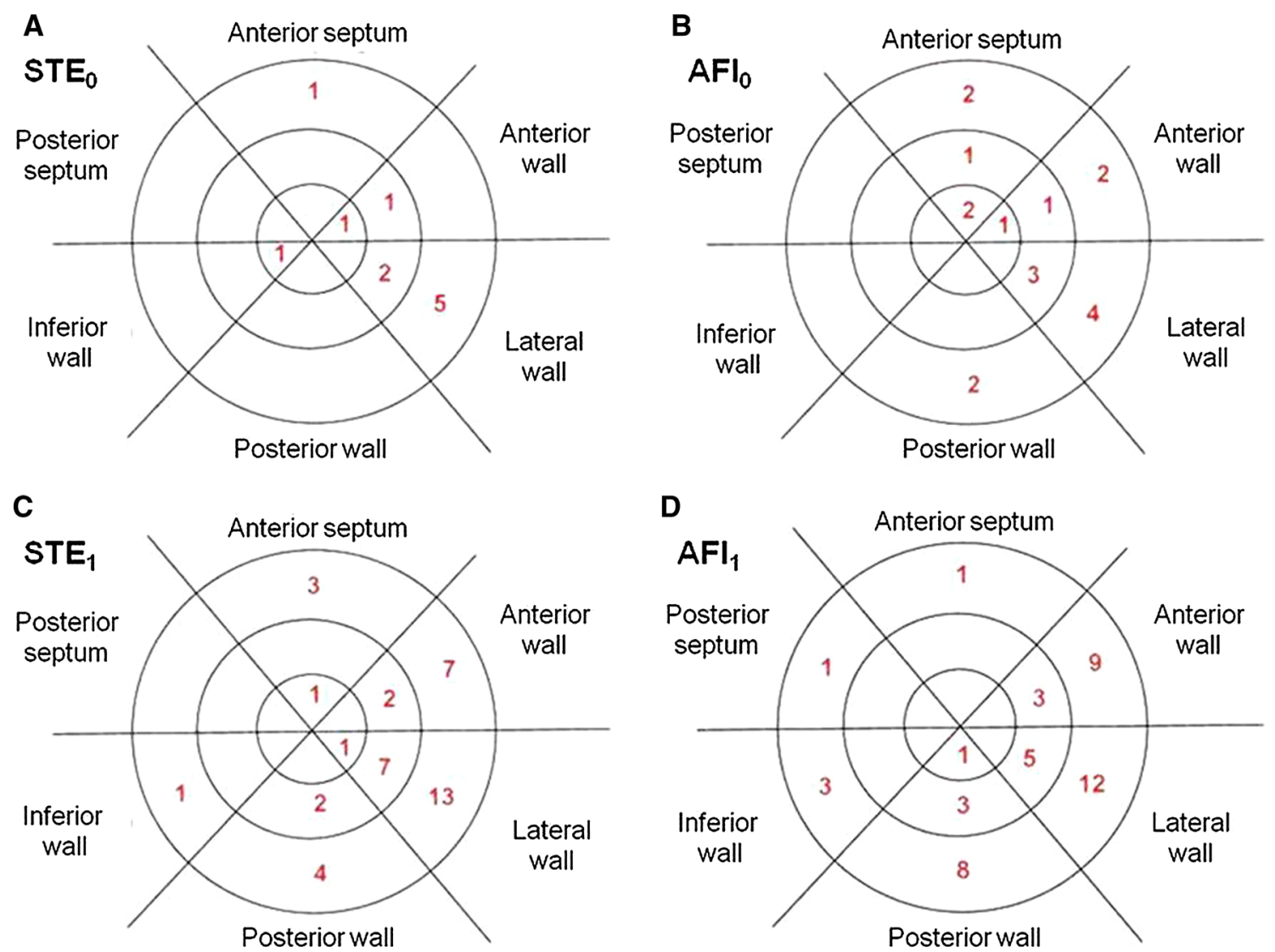

Fig. 2 The number of segments excluded from analysis by STE and AFI. Polar maps display regional dispersion of feasibility-with sum of 45 excluded segments in both stages of DSE in the region of anterior, lateral and posterior wall in analysis using STE versus 7 segments in region of septum and inferior wall. From AFI this proportion was similar with 54 versus 10 excluded segments in

the setting of stress test [15-17]. Additional concerns were evoked by JUSTICE study indicating lower than expected reproducibility of deformation measurements when compared among different vendors of echocardiographic machines [18]. On the other hand, recently published studies documented the advantage of quantitative assessment of deformation over visual evaluation of regional wall motion of left ventricle and good reproducibility of strain/strain rate parameters at consecutive levels of low-dose dobutamine protocol $[19,20]$.

In our study we documented the moderate clinical utility of standard 2D STE for the evaluation of left ventricular function during DSE with high percentage of segments amenable to quantification $(>90 \%)$ but with limited interobserver agreement, especially when systolic longitudinal strain was measured during significant tachycardia (mean value of heart rate $139 \pm 17$ beat per minute) at peak stage of test. The high observed feasibility of STE and AFI is comparable to values reported in the literature although the findings in our study may be influenced by

respective regions of left ventricle. $S T E_{O}$ number of segments excluded from strain measurements by $\mathrm{STE}$ at rest, $A F I_{O}$ number of segments excluded from strain measurements by AFI at rest, $S T E_{l}$ number of segments excluded from strain measurements by STE at peak stress test, $A F I_{1}$ number of segments excluded from strain measurements by AFI at peak stress test

preselection of the subjects with all segments suitable for visual assessment [21, 22].

The novel, simplified AFI technique offered similar feasibility, lower coefficient of variance both during rest and peak stress stage (8.7 vs $13.3 \%$ and 16 vs $24.2 \%$ respectively) and significantly (about two times) shorter time needed for analysis. The preponderance of AFI over velocity vector imaging (VVI) -based strain calculations was observed in earlier studies concerning patients with suspected or documented coronary artery disease and using magnetic resonance strain imaging as a golden standard [23].

As expected the localization of segments excluded from analysis was similar for both traditional STE and AFI with lower feasibility in regions of anterior, lateral and posterior wall (see polar maps in Fig. 2) [24].

Despite worse feasibility and repeatability during peak stage of DSE, a highly significant strong correlation between systolic longitudinal strains measured by both methods was preserved not only at rest but also during peak stage of the test (see Figs. 3, 5). Good agreement between 

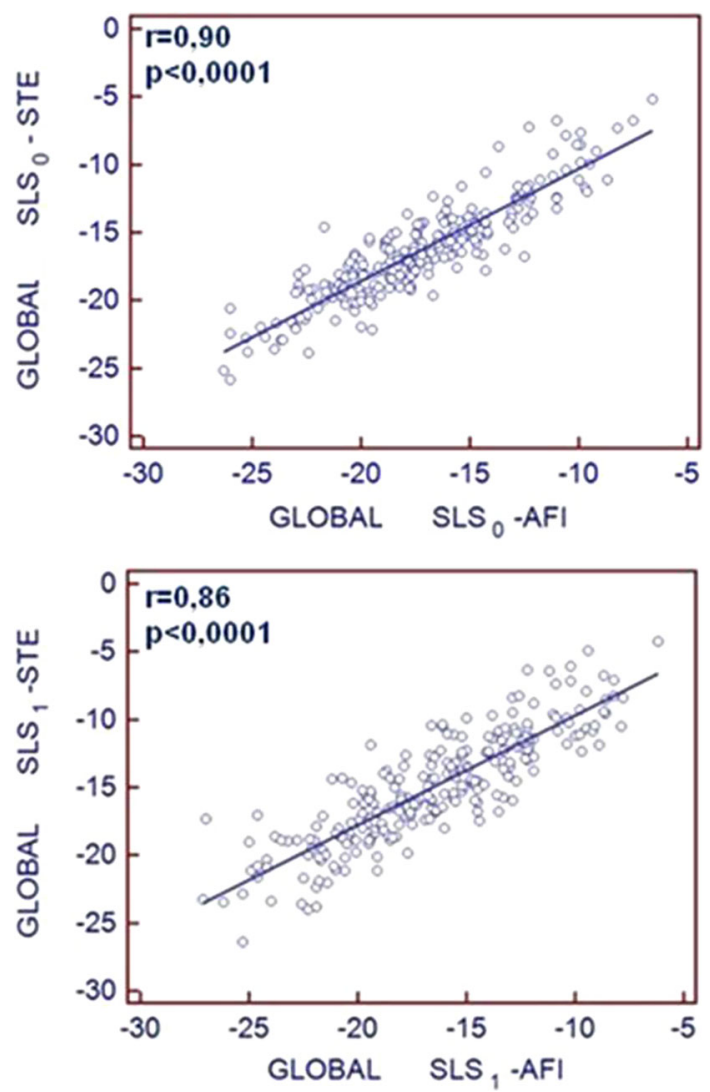

Fig. 3 The graphs presenting the significant correlation between systolic longitudinal strain measured by classic speckle tracking echocardiography (STE) and automated function imaging (AFI) during baseline (upper panel, index 0) and at peak stage of DSE (lower panel, index 1)

both techniques for global left ventricular strain evaluation was also corroborated by Bland-Altman analysis (Fig. 4).

The present clinical standard for contractility evaluation during echocardiographic stress test is visual assessment of wall motion (endocardial displacement and myocardial thickening) performed optimally as expert consensus by experienced and accordingly trained observers and supported by second harmonic imaging, echocardiographic contrast enhancement as needed, digital storage and quad screen format $[6,25,26]$. The visual assessment is yet limited by high interobserver variability and relies mainly on myocardial radial performance, whereas subendocardial longitudinal myocardial layer is the most sensitive to ischemia.

Considering the known limitation of visual assessment of wall motion during echocardiographic stress test, especially those connected with significant tachycardia (such as dobutamine, exercise and rapid pacing) and meticulous analysis necessary in standard STE, the novel AFI method appears faster, offering user-friendly polar maps of the left ventricle and thus a potential to become a useful clinical tool.
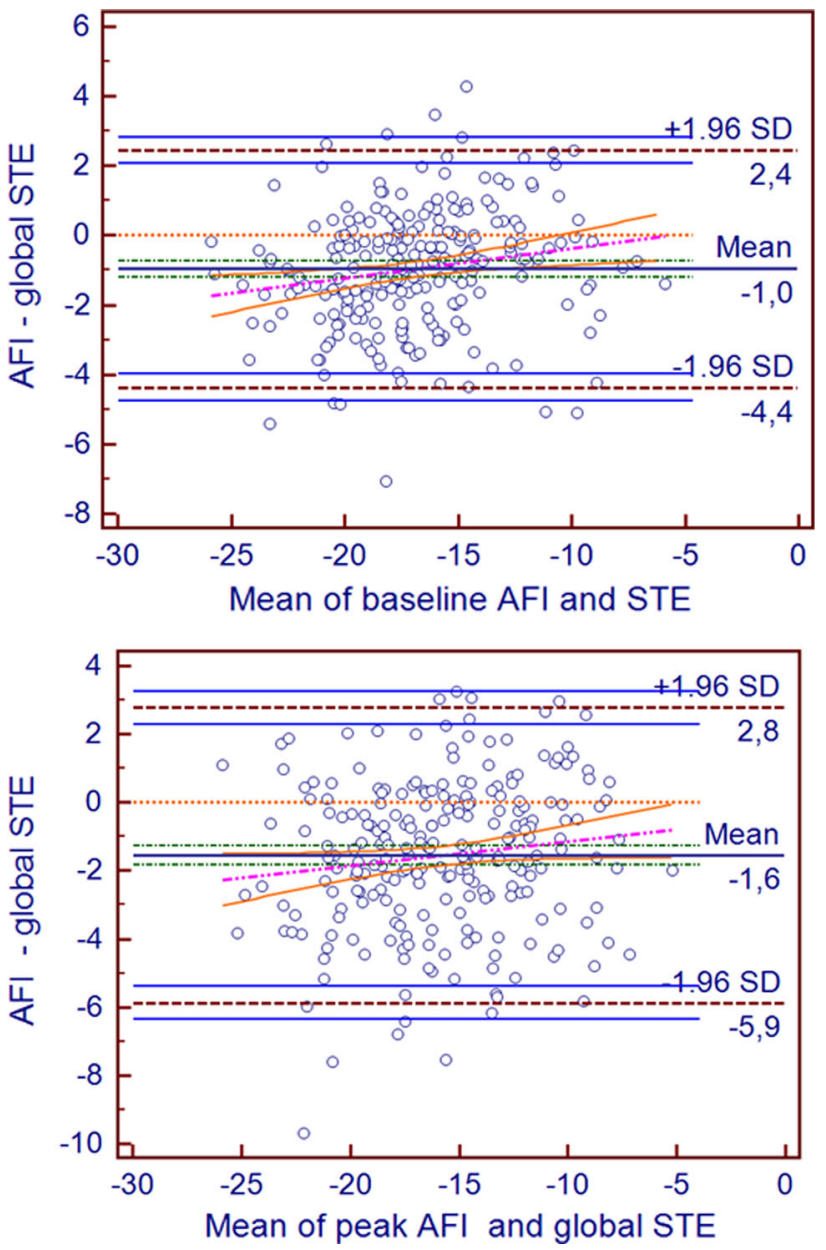

Fig. 4 The Bland-Altman analysis of global systolic longitudinal strain measurements achieved by both methods at baseline (upper panel) and peak stage. Dotted orange line-line of equality, pink line-regression line, orange continuous lines-confidence intervals of regression line, green lines-confidence intervals of mean of difference line, blue lines - confidence intervals of limit of agreement lines

\section{Limitations}

The main limitation of our study is a lack of a golden standard for echocardiographic measures of deformation. Magnetic resonance imaging myocardial tagging could be proposed as a reference but its practical use is very limited. Instead, we focused on a direct comparison of two easily amenable echocardiographic modalities.

Another limitation of our study is the exclusion of subjects with the suboptimal echocardiographic visualization assessment of endocardial border. Nevertheless the percentage of subjects excluded since suboptimal quality of visualization was really low (below $3 \%$ ) and the number of included examination still exceeded 200 DSE tests with sum $>4,000$ analyzed segments. 
Fig. 5 Correlations of peak regional longitudinal strain values measured by STE and AFI during baseline and peak DSE. $S L S_{O}$ systolic longitudinal strain at baseline, $S L S_{1}$ systolic longitudinal strain at peak stage of dobutamine test. STE speckle tracking echocardiography, $A F I$ automated function imaging. Segment 17- mid segment of anterior septum, 5-mid segment of lateral wall, 8- mid segment of inferior wall
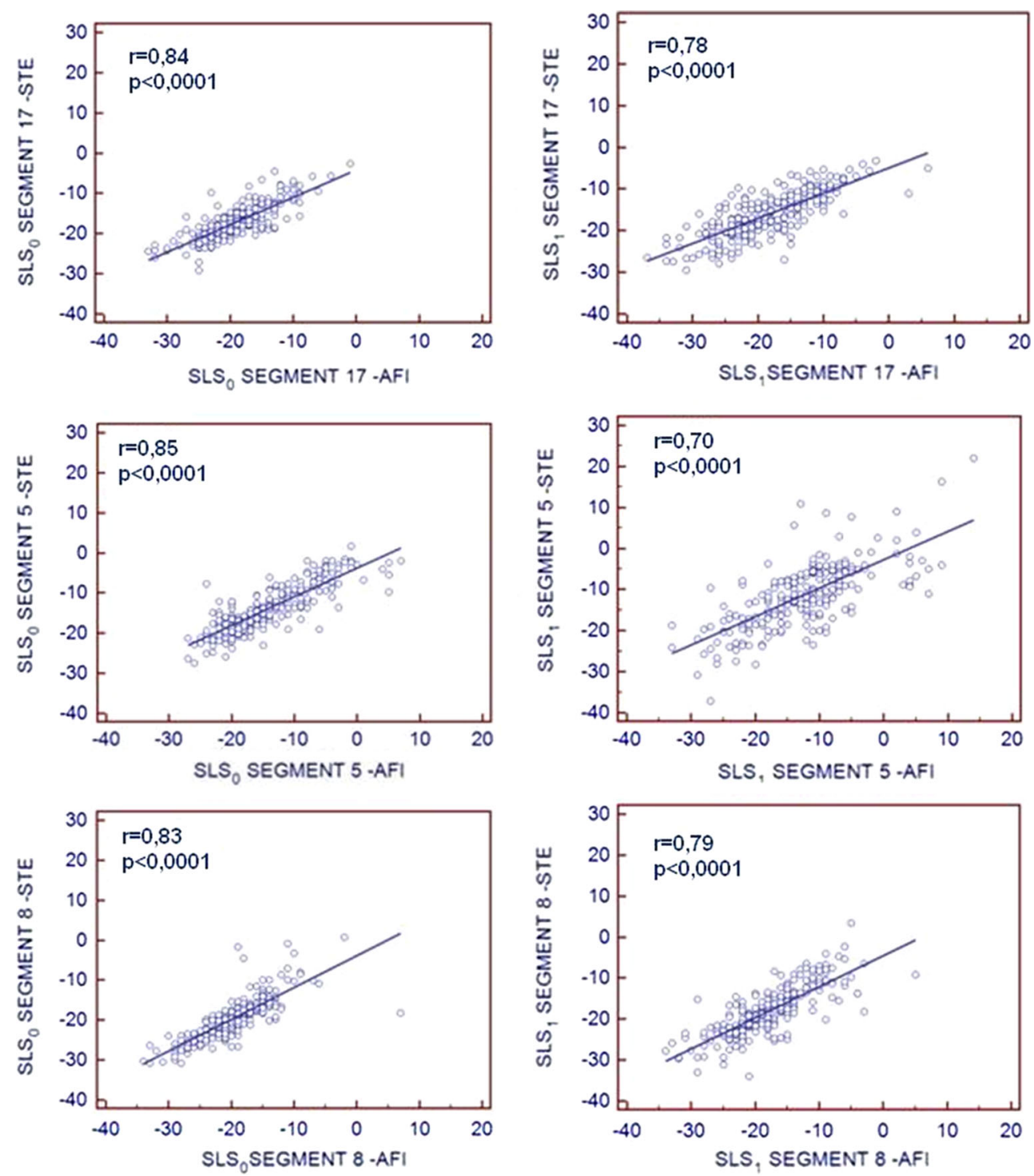

Single-center character and the assessment of STE and AFI correlation confined to a single vendor of echocardiographic equipment may be considered as subsequent limitation of our study as evidence is growing regarding a poor agreement between different STE softwares.

\section{Conclusions}

The quantitative assessment of longitudinal regional and global strain during DSE is feasible by both standard 2D STE and AFI methods. Parameters achieved by those techniques showed strong correlations at rest ant at the peak stage of dobutamine stress test. The shorter time necessary for analysis by AFI method and lower operator-dependency confirmed by improved interobserver agreement indicate potential utility of AFI for objective evaluation of left ventricular function during echocardiographic stress test.

Acknowledgments The study was supported from the State Committee for Scientific Research Grant-N N402 500240.

\section{Conflict of interest None.}

Open Access This article is distributed under the terms of the Creative Commons Attribution License which permits any use, distribution, and reproduction in any medium, provided the original author(s) and the source are credited. 


\section{References}

1. Pellikka PA, Nagueh SF, Elhendy AA, Kuehl CA, Sawada SG (2007) American Society of Echocardiography recommendations for performance, interpretation and application of stress echocardiography. J Am Soc Echocardiogr 20:1021-1041

2. Sicari R, Nihoyannopoulos P, Evangelista A, Kasprzak J, Lancellotti P, Poldermans D et al (2008) EAE Guidelines Stress echocardiography expert consensus statement European Association of Echocardiography (EAE) (a registered branch of the ESC). Eur J Echocardiogr 9:415-437

3. Gianrossi R, Detrano R, Mulvihill D, Lehmann K, Dubach P, Colombo A et al (1989) Exercise-induced ST depression in the diagnosis of coronary artery disease. Meta-anal Circ 80:87-98

4. Kwok Y, Kim C, Grady D, Segal M, Redberg R (1999) Metaanalysis of exercise testing to detect coronary artery disease in women. Am J Cardiol 83:660-666

5. Madler CF, Payne N, Wilkenshoff U, Cohen A, Derumeaux GA, Pierard LA et al (2003) For the MYDISE Study Investigators (Myocardial Doppler in Stress Echocardiography). Non-invasive diagnosis of coronary artery disease by quantitative stress echocardiography: optimal diagnostic models using off-line tissue Doppler in the MYDISE study. Eur Heart J 24:1584-1594

6. Kasprzak JD, Paelinck B, Ten Cate FJ, Vletter WB, de Jong N, Poldermans D et al (1999) Second harmonic imaging improves the visualization of left ventricular endocardial border. A comparison of native and contrast-enhanced studies. Am J Cardiol 83:211-217

7. Wijns W, Kolh P, Danchin N, Di Mario C, Falk V, Folliguet T et al (2010) The task force on myocardial revascularization of the European society of cardiology (ESC) and the European association for cardio-thoracic surgery (EACTS) developed with the special contribution of the European association for percutaneous cardiovascular interventions (EAPCI) ESC/EACTS guidelines on myocardial revascularisation. Eur Heart J 31:2501-2555

8. Lipiec P, Wejner-Mik P, Krzemińska-Pakuła M, Kuśmierek J, Płachcińska A, Szumiński R et al (2008) Accelerated stress realtime myocardial contrast echocardiography for the detection of coronary artery disease: comparison with $99 \mathrm{mTc}$ single photon emission computed tomography. $\mathrm{J}$ Am Soc Echocardiogr 21:941-947

9. Geyer H, Carraciolo G, Abe H, Wilansky S, Carerj S, Gentile F et al (2010) Assessment of myocardial mechanics using speckle tracking echocardiography: fundamentals and clinical applications. J Am Soc Echocardiogr 23:351-369

10. Urheim S, Edvardsen T, Trop H, Angelsen B, Smiseth OA (2000) Myocardial strain by doppler echocardiography: validation of a new method to quantify regional myocardial function. Circulation 102:1158-1164

11. Kowalski M, Herregods MC, Herbots L, Weidemann F, Simmons L, Strotmann J et al (2003) The feasibility of ultrasonic regional strain and strain rate imaging in quantifying dobutamine stress echocardiography. Eur J Echocardiogr 4:81-91

12. Voigt J-U, Nixdorff U, Bogdan R, Exner B, Schmiedehausen K, Platsch G et al (2004) Comparison of deformation imaging and velocity imaging for detecting regional inducible ischaemia during dobutamine stress echocardiography. Eur Heart J 25:1517-1525

13. Kukulski T, Jamal F, Herbots L, D'hooge J, Bijnens B, Hatle L et al (2003) Identification of acutely ischemic myocardium using ultrasonic strain measurements. A clinical study in patients undergoing coronary angioplasty. J Am Coll Cardiol 41:810-819

14. Perk G, Tunick PA, Kronzon I (2007) Non-doppler two-dimensional strain imaging by echocardiography-from technical considerations to clinical applications. J Am Soc Echocardiogr 20:234-243

15. Zhang L, Gao J, Xie M, Yin P, Liu W, Li Y, et al. (2013) Left ventricular three-dimensional global systolic strain by real-time three-dimensional speckle-tracking in children: feasibility, reproducibility, maturational changes, and normal ranges. J Am Soc Echocardiogr. doi: 10.1016/j.echo.2013.05.002

16. Altiok E, Neizel M, Tiemann S, Krass V, Kuhr K, Becker M et al (2012) Quantitative analysis of endocardial and epicardial left ventricular myocardial deformation-comparison of strain-encoded cardiac magnetic resonance imaging with two-dimensional speckle-tracking echocardiography. J Am Soc Echocardiogr 25:1179-1188

17. Belghiti H, Brette S, Lafitte S, Reant P, Picard F, Serri K et al (2008) Automated function imaging: a new operator-independent strain method for assessing left ventricular function. Arch Cardiovasc Dis 101:163-169

18. Takigiku K, Takeuchi M, Izumi C, Yuda S, Sakata K, Ohte N et al (2012) Normal range of left ventricular 2-dimensional strain-Japanese ultrasound speckle tracking of left ventricle (JUSTICE) study. Circ J 76:2623-2632

19. Anwar A, Nosir Y, Alasnag M, Llemit MA, Elhagoly AA, Chamsi-Pasha H (2013) Quantification of left ventricular longitudinal strain by two-dimensional speckle tracking: a comparison between expert and non-expert readers. Int J Cardiovasc Imaging 29:1451-1458

20. Elmayergi NH, Goodman JM, Lee LS, Sasson Z (2013) Are measures of left ventricular systolic performance during low dose dobutamine stress echocardiograms repeatable over time? Int J Cardiovasc Imaging 29:1281-1286

21. Anwar AM (2012) Global and segmental myocardial deformation by $2 \mathrm{D}$ speckle tracking compared to visual assessment. World $\mathrm{J}$ Cardiol 4:341-346

22. Chrzanowski Ł, Lipiec P, Krzemińska-Pakuła M, Wejner-Mik P, Jasińska A, Drożdż J et al (2007) Echokardiograficzna ocena odkształcenia lewej komory przy zastosowaniu techniki doplera tkankowego oraz śledzenia markerów akustycznych (speckle tracking). Pol J Cardiol 9:195-202

23. Bansal M, Cho GY, Chan J, Leano R, Haluska BA, Marwick TH (2008) Feasibility and accuracy of different techniques of twodimensional speckle based strain and validation with harmonic phase magnetic resonance imaging. J Am Soc Echocardiogr 21:1318-1325

24. Marwick T, Leano RL, Brown J, Sun J-P, Hoffman R, Lysansky $P$ et al (2009) Myocardial strain measurement with 2-dimensional speckle tracking echocardiography. Definition of normal range. J Am Coll Cardiol Imaging 2:80-84

25. Picano E, Lattanzi F, Orlandini A, Marini C, L'Abbate A (1991) Stress echocardiography and the human factor: the importance of being expert. J Am Coll Cardiol 17:666-669

26. Anand DV, Theodosiadis ID, Senior R (2004) Improved interpretation of dobutamine stress echocardiography following 4 months of systematic training in patients following acute myocardial infarction. Eur J Echocardiogr 5:12-17 